\title{
Experimental Study on Stratification Morphology of the Molten Pool During Severe Accident
}

\author{
Yang Li, Houjun Gong*, Yunwen Hu, Shengxing Yang, Yong Li and Yuanfeng Zan \\ CNNC Key Laboratory on Nuclear Reactor Thermal Hydraulics Technology, Nuclear Power Institute of China, Chengdu, China
}

\section{OPEN ACCESS}

Edited by:

Luteng Zhang,

Chongaing University, China

Reviewed by:

Yapei Zhang,

X'an Jiaotong University, China

Yan Xiang,

Royal Institute of Technology, Sweden

*Correspondence:

Houjun Gong

ghitsing@126.com

Specialty section:

This article was submitted to

Nuclear Energy,

a section of the journal

Frontiers in Energy Research

Received: 11 November 2021 Accepted: 23 November 2021

Published: 24 December 2021

Citation:

$L i Y$, Gong $H, H u Y$, Yang $S, L i Y$ and Zan $Y$ (2021) Experimental Study on Stratification Morphology of the Molten

Pool During Severe Accident.

Front. Energy Res. 9:813298.

doi: 10.3389/fenrg.2021.813298
Stratification morphology of a molten pool under severe reactor accident was investigated by the CESEF experimental facility. The experimental scale was 5,000 $\mathrm{g}$, the atomic ratio of $\mathrm{U} / \mathrm{Zr}$ was 1.5, the content of stainless steel was 10\%, and the oxidation degree of Zr was 40-100\%. It was shown that the molten pool was obviously stratified within the range of experimental parameters; one was a metal layer, and the other was an oxide layer. The layered morphology of the molten pool was different with the composition of different corium. With the decrease in the Zr oxidation degree, the metal layer moved downward in the molten pool, and the molten pool would overturn. The main elements in the oxide layer were $\mathrm{U}, \mathrm{Zr}$, and $\mathrm{O}$, and the content of stainless steel was low. The main element in the metal layer was stainless steel and contained a certain amount of $U$ and $Z r$.

Keywords: stratification morphology, prototype material, molten pool, severe accident, experimental

\section{INTRODUCTION}

In the severe accident of a nuclear reactor, such as the Fukushima Daiichi NPP (nuclear power plant), the fuel and structural materials in the core had been melted (Report of Japanese Government to the IAEA Ministerial Conference on Nuclear Safety, 2011). The molten materials $\left(\mathrm{UO}_{2}, \mathrm{Zr}, \mathrm{ZrO}_{2}\right.$, stainless steel, etc.) were heated up and melted by decay heat as the coolant in the pressure vessel was depleted. Finally, a molten pool with $\mathrm{U}-\mathrm{Zr}-\mathrm{Fe}-\mathrm{O}$ as the main element is formed. The configuration of the molten pool, so called corium, as it affects the failure mechanism of the pressure vessel. The thermodynamic properties of the melt determine that the molten pool will be stratified at the lower head of the pressure vessel, and the stratification morphology of the molten pool determines the heat distribution of the lower head, which is one of the key factors of IVR (retention in the melt stack) strategy. For a long time, the stratification form of the molten pool has been a hot and difficult point in the field of severe accidents at home and abroad.

It was suggested that the compositions of the molten pool formed at the late phase of a severe accident would be in the domain of the miscibility gap of the $\mathrm{U}-\mathrm{Zr}-\mathrm{Fe}-\mathrm{O}$ phase diagram, and two immiscible liquid phases will be formed (Theofanous et al., 1997). Edwards (Edwards and Martin, 1966), Hirotomo (Ikeuchi et al., 2020), Guéneau (Guéneau et al., 1998), Bakardjieva (Bakardjieva et al., 2010), Pierre Yves (Chevalier et al., 2004), and Pascal Piluso (Pascal et al., 2005) found that the U-O binary system will be divided into two layers, and Guéneau (Guéneau et al., 1998), Jinho Song (Song et al., 2020), and Bechta (Bechta and Granovsky, 2008) found that the U-Zr-O ternary system will also be divided into two layers. The thermodynamic database of the $\mathrm{U}-\mathrm{Zr}-\mathrm{Fe}-\mathrm{O}-\mathrm{B}-\mathrm{C}-$ FPs complex system is established by the SGTE (scientific group thermodata Europe) database (Fukasawa et al., 2005), and the calculated results are consistent with the experimental data of MASCA (Fukasawa and Shigeyuki, 2012). Bechta (Bechta et al., 2005) supplemented and revised the European nuclear database (NUCLEA) with the help of the experimental data of CORPHAD 
and METCOR projects, providing a more accurate model for the thermodynamic calculation of the melt.

The RASPLAV project (Asmolov and Behbahani, 2000; Bechta and Khabensky, 2001; Bechta and Khabensky, 2006) carried out experimental research with prototype melt materials and found that the bottom of the molten pool was rich in $\mathrm{U}$ and the top was rich in Zr. In the MASCA project (Asmolov, 2004; Tsurikov et al., 2007), three different melting pool stratification forms were found, namely, the molten pool with the metal layer above and the oxide layer below, the molten pool with the metal layer below and the oxide layer above, and the molten pool with the metal layer suspended in the oxide layer. The South Korea COSMOS project (Kang and Park, 2014) found that the molten pool presents a three-layered structure, with a metal layer in the upper part and an oxide layer and some heavy metals in the lower part. The EU IVMR project (Almjashev et al., 2018) studied the layered inversion phenomenon of the molten pool and also found the stratification morphology of the threelayered molten pool. Masahide Takano (Takano et al., 2014) and other experiments on the Fukushima Daiichi nuclear accident show that the molten pool is divided into a metal layer ( $\mathrm{Fe}-\mathrm{Cr}-\mathrm{Ni}$ U-B-C) and an oxide layer (U-Zr-O). Sang Mo (Sang et al., 2016) also carried out experimental research on the prototype melt materials for the Fukushima Daiichi nuclear accident and found that the molten pool was divided into two layers, with a metal layer at the top and an oxide layer at the bottom.

In this study, the configuration of the molten pool for a typical Hualong nuclear reactor composition was investigated by a melting and solidification experiment using an induction heating technique in a cold crucible. The physical and chemical analyses were conducted for the samples taken from the solidified corium ingot to investigate the morphology and chemical composition at each position.

\section{EXPERIMENTAL METHODS}

\section{Experimental Setup}

The CESEF facility is designed to investigate the stratification of the molten pool at the lower head of the pressure vessel under severe accident, which adopts an electromagnetic induction heating technique with a cold crucible. This technique has been optimized for melting of various reactor materials ranging from a metallic mixture of $\mathrm{Zr}$ and stainless steel to an oxidic mixture of $\mathrm{UO}_{2}$ and $\mathrm{ZrO}_{2}$, which are the prototypic reactor materials (Sang et al., 2016). The cold crucible consists of a copper plate and an induction coil. The copper plate is forced to circulate to provide cooling. The induction coil is located to provide induction heating by using a high frequency generator. A high frequency generator was designed to supply power up to $400 \mathrm{~kW}$ with $100 \mathrm{kHz}$. However, the frequency could change depending on several factors, such as crucible dimensions, induction coil turns, and melting materials, and so on (Sang et al., 2016). The cold crucible is installed in the inerting chamber and has a diameter of $110 \mathrm{~mm}$ and a height of $170 \mathrm{~mm}$. A schematic diagram of the facility is given in Figure 1. There is a window at the top of the inerting chamber to measure the temperature of the molten pool, which adopts two-color infrared. The temperature measurement rang of two-color infrared is $1,500-3,500^{\circ} \mathrm{C}$, and the measurement accuracy is $\pm 1.5 \%$. Argon gas is purged into the inerting chamber through a tube at the bottom of the inerting chamber.

Figure 2 shows the melt generation behavior along with the supplied voltage. The voltage increased by $10-20 \mathrm{~V}$ every 2 minutes. When the voltage was about $140-160 \mathrm{~V}$, the $\mathrm{Zr}$ ring started to light up. After $200 \mathrm{~V}$, the molten pool started to melt, the current decreased, and the power decreased. It was held for 2 $h$, and the experiment was ended.

\section{Experimental Materials}

The cold crucible was filled with a mixture of $\mathrm{UO}_{2}$ pellets, $\mathrm{ZrO}_{2}$ powder, $\mathrm{Zr}$ pellets, and stainless steel pellets. The composition of the prototype material is usually defined by the atomic molar ratio $\left(\mathrm{R}_{\mathrm{U} / \mathrm{Zr}}\right)$ of $\mathrm{U}$ and $\mathrm{Zr}$, the oxidation ratio $(\mathrm{Cn})$ of $\mathrm{Zr}$, and the mass ratio $\left(\mathrm{X}_{\mathrm{SS}}\right)$ of stainless steel to the prototype material. Assuming that the mass of each component in the prototype material is $\mathrm{m}_{\mathrm{i}}, \mathrm{i}=\mathrm{UO}_{2}, \mathrm{ZrO}_{2}, \mathrm{Zr}$, and SS (stainless steel), the calculation method of each parameter is as follows:

$$
\begin{gathered}
R_{U / Z r}=\frac{m_{U O_{2}} / 271}{m_{\mathrm{ZrO}_{2}} / 123+m_{\mathrm{Zr}} / 91}, \\
C_{n}=\frac{m_{\mathrm{ZrO}_{2}} / 123}{m_{\mathrm{ZrO}_{2}} / 123+m_{\mathrm{Zr}} / 91}, \\
X_{S S}=\frac{m_{S S}}{m_{\mathrm{UO}_{2}}+m_{\mathrm{ZrO}_{2}}+m_{\mathrm{Zr}}+m_{S S}} .
\end{gathered}
$$

The experimental scale is $5,000 \mathrm{~g}$, the $\mathrm{R}_{\mathrm{u} / \mathrm{Zr}}$ is 1.5 , the $\mathrm{X}_{\mathrm{SS}}$ is $10 \%$, and the $\mathrm{Cn}$ is $40-100 \%$ experimental conditions are shown in Table 1. Cn of condition one is $100 \%$, Cn of condition two is $80 \%$, and $\mathrm{Cn}$ of condition three is $40 \%$. The loading process is as follows: first, a layer of $\mathrm{ZrO}_{2}$ was laid at the bottom of the crucible to protect the crucible during the melting process. Then, $\mathrm{UO}_{2}$ pellets and $\mathrm{ZrO}_{2}$ powder were added into the crucible; stainless steel was placed in the middle of the crucible to prevent direct contact between stainless steel and the crucible wall. It was allowed to flatten and compact; then, a layer of ring composed of $\mathrm{Zr}$ pellets was laid on the upper part as an initiator for induction heating, and finally, a layer of $\mathrm{ZrO}_{2}$ powder was laid on the upper part to reduce heat loss from the melt to the environment. Some holes were initially made by the stainless steel wire for gas venting.

\section{Chemical Analysis}

After the experiment, the molten ingot was cut from the middle by using a wire cutting machine and then sampled at different positions by using a tungsten cobalt hollow drill. The samples are used to analyze the chemical composition and structure, which was obtained from X-ray fluorescence (XRF) and a scanning electron microscope/energy dispersive spectrometer (SEM/EDS). The XRF equipment is S8 TIGER, a wavelength-dispersive X-ray fluorescence spectrometer produced by the BROOKE Company, with a maximum excitation current of $170 \mathrm{~mA}$ and a maximum excitation point of $60 \mathrm{kV}$. The SEM equipment is Gemini SEM, a fully functional emission scanning electron microscope produced 


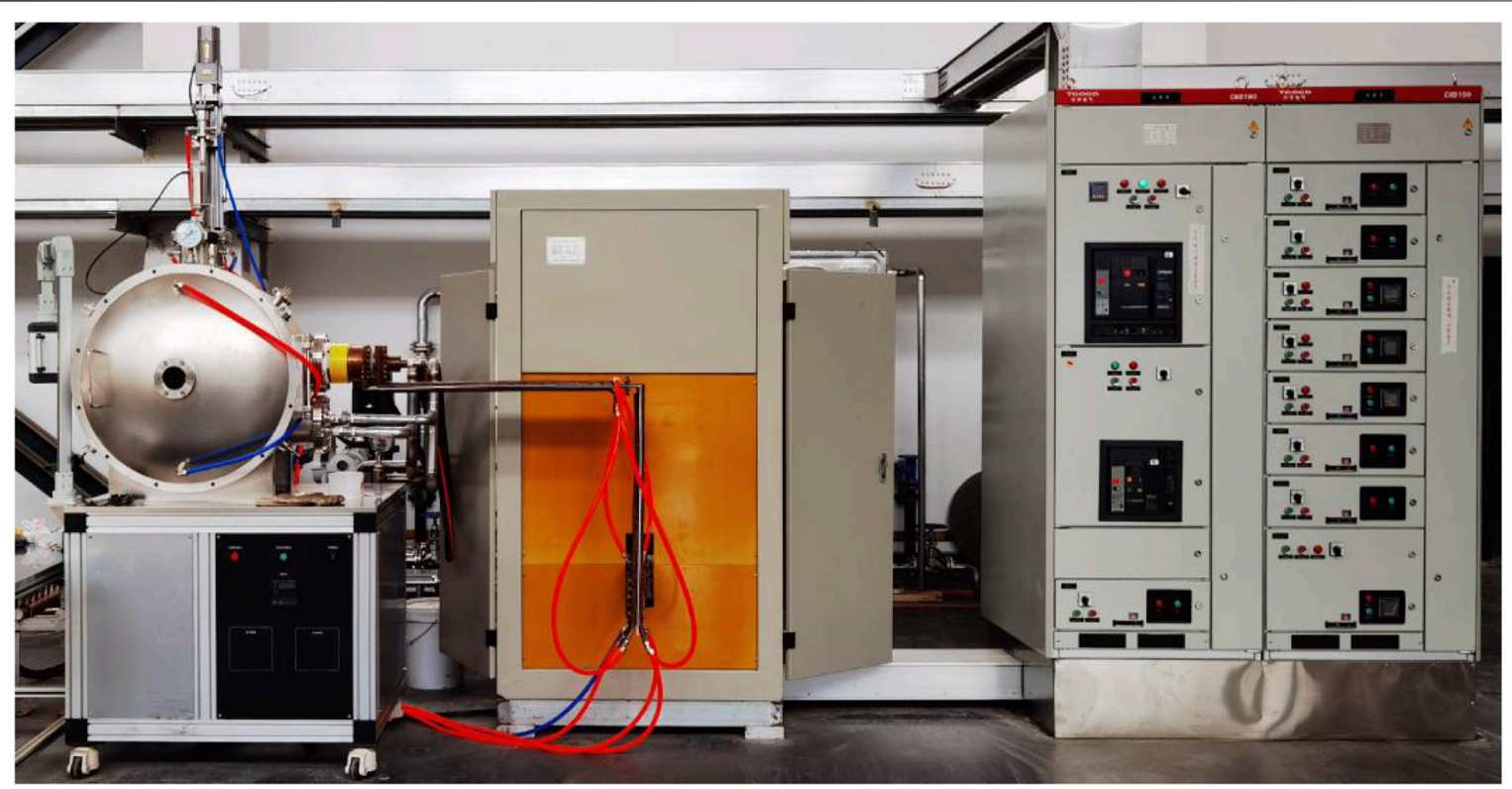

FIGURE 1 | Schematic diagram of the CESEF facility.

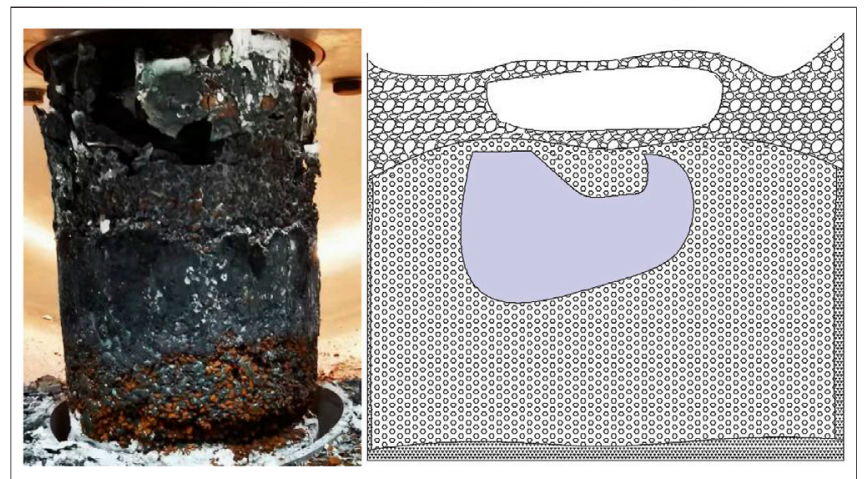

FIGURE 2 | Morphology of the corium ingot after the experiment (2).

by the Zeiss Company, and equipped with two Oxford X-ray spectrometers, which can be widely used for micro area morphology and composition analysis.

\section{RESULTS AND DISCUSSION}

\section{Morphology of Corium Ingot}

Figure 2 shows the morphology of the corium ingot after the experiment. It is shown that the corium ingot consists of six parts; the upper crust, upper cavity, metal-rich layer, oxide-rich layer, side crust, and lower unmelted part. The upper crust is mainly unmelted $\mathrm{ZrO}_{2}$ and sintered melt during the melting process (Sang et al., 2016) because the top layer is exposed to the atmosphere and cooled by radiating heat to the inerting chamber, so that the top layer has been cooled before reaching the melting temperature. Some amount of the unmelted layer was observed at the bottom and side of the corium ingot. A large cavity is formed between the upper crust and the molten ingot, which is formed due to the volume reduction of molten particles after melting and the downward collapse of the molten pool (Song et al., 2018). A lump of the corium ingot below the cavity was found; the metal layer and oxide layer are easy to distinguish after cut from the middle by the wire-cutting machine. The metal layer is bright, and the oxide layer is dark. After separating the metal layer and oxide layer, it is found that the metal layer is very hard and the oxide layer is brittle. It can be considered that liquid phase separation occurs in the melting process because phase separation will not be so complete if it occurs in the solidification process (Sang et al., 2016). The density of the metal layer and oxide layer is different so as to form a different morphology of the molten pool. We found three different molten pool stratification: the molten pool with a metal layer above and an oxide layer below, the molten pool with a metal layer below and an oxide layer above, and the molten pool with the metal layer suspended in the oxide layer, which is similar to the experimental results of the MASCA project (Asmolov, 2004; Tsurikov et al., 2007). A side crust with a thickness of about $2 \mathrm{~mm}$ is formed between the ingot and crucible. The crust isolates the internal $2,800^{\circ} \mathrm{C}$ high-temperature melt from the cold crucible, which shows that the thermal conductivity of the crust of the melt is very small.

\section{Stratification Morphology of the Molten Pool}

Figure 3 is a longitudinal sectional view of the corium ingot. The metal layer and oxide layer in the ingot are very easy to distinguish. The metal layer is bright, and the oxide layer is dark. In working condition 1, the melt composition is mainly oxide and $\mathrm{Zr}$ metal is completely oxidized. No obvious stratification is found in the molten pool formed by this melt 

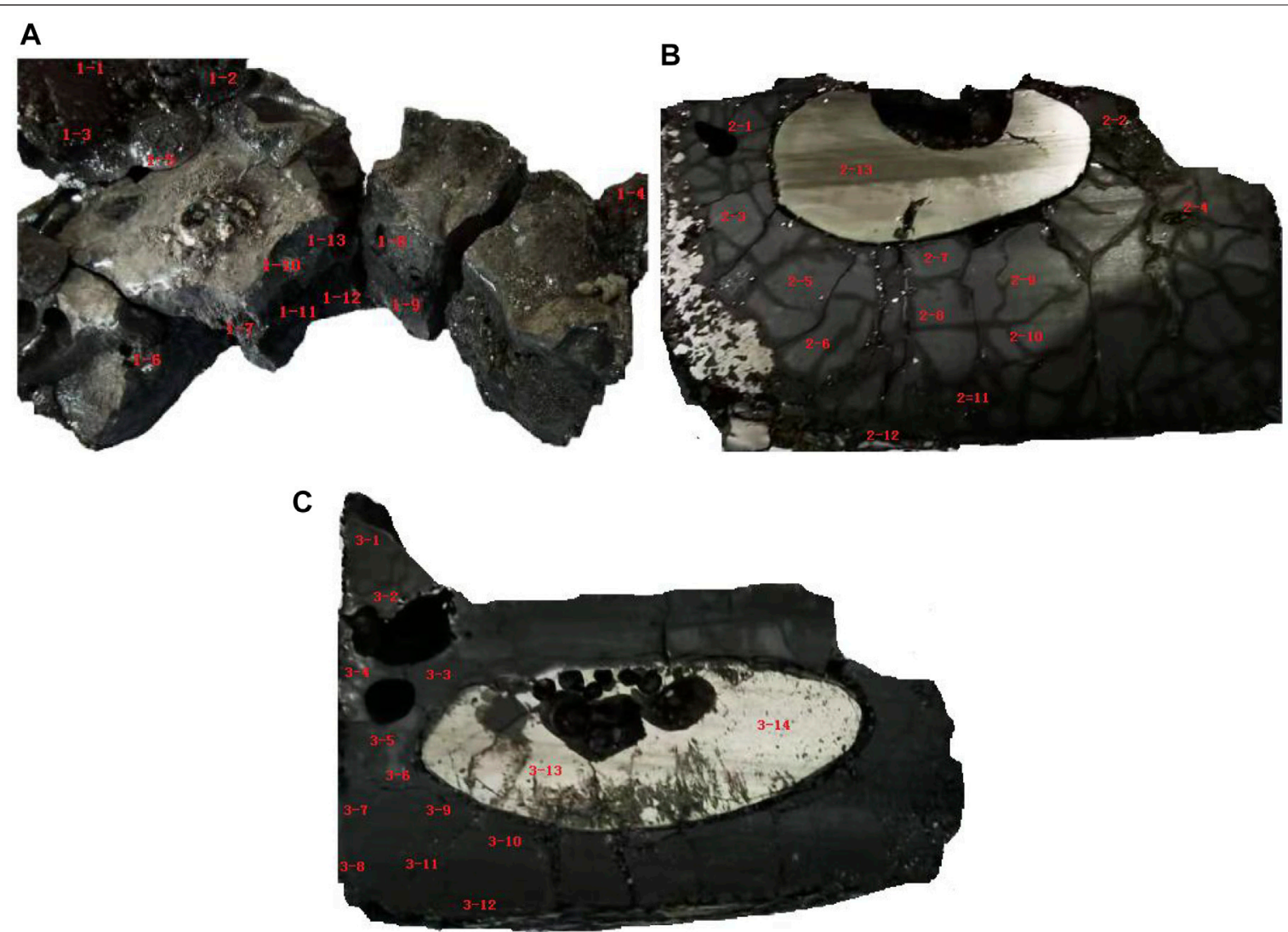

FIGURE 3 | Longitudinal sectional view of the corium ingot (A) 1, (B) 2, (C) 3.

composition, and only a metal ball with a diameter of about $10 \mathrm{~mm}$ is formed at the top of the molten pool. With the decrease in the $\mathrm{Zr}$ oxidation degree, obvious stratification began to appear in the molten pool, forming a bright metal layer and dark oxide layer. In working condition 2 , the formed metal layer is located in the upper part of the molten pool, and the oxide layer is located in the lower part of the molten pool. The metal layer formed in working condition three is located in the middle of the molten pool; the oxide layer wraps the metal layer, and the approximate metal layer is suspended in the middle of the molten pool. The MA experiment in the MASCA project also found three-layered molten pools (Tsurikov et al., 2007).It is found that the metal ball in condition one is mainly composed of stainless steel, which will be described in detail in section 3.3 composition analysis. The density of stainless steel is lower than that of the oxide layer, and it floats on the upper part of the oxide layer. As the oxidation degree of $\mathrm{Zr}$ decreases, more $\mathrm{U}$ and $\mathrm{Zr}$ elements enter the metal layer, the density of the metal layer gradually increases, and the metal layer begins to migrate downward. By working condition 3, the density of the metal layer has been equivalent to that of the oxide layer. The metal layer is suspended in the oxide layer.

\section{Composition Analysis}

The corium ingot is cut axially, and then, a small part of the ingot was sampled at different positions, including the upper crust, around the cavity, side crust, lower crust, metal layer, and oxide layer, as shown in Figure 4.

Elemental compositions of the samples taken from the different positions of the corium ingot are shown in Table 2 . Table 2 only shows the XRF analysis results of condition 1 . Total concentrations of $\mathrm{U}, \mathrm{Zr}, \mathrm{Fe}, \mathrm{Ni}$, and $\mathrm{Cr}$ in the samples were less than $100 \%$, which implies that the samples contain other elements such as oxygen that are not measurable by XRF. In condition $1,1-1$ to $1-5$ are the sampling points of the upper crust. It is found that the content of elements in different positions of the upper crust varies greatly, mainly because on the one hand, the top melt is not completely melted and is only sintered together, so it is difficult for mass transfer in the crust. On the other hand, after the upper crust is formed, it is quickly separated from the lower molten pool, forming a large cavity, and then, mass transfer will not occur with the molten pool. On the other hand, at high temperature, some elements will escape from the molten pool and then be adsorbed on the upper crust. Three reasons lead to great differences in the distribution of elements at different positions of the upper crust, and other conditions also have similar characteristics.

The main elements of the oxide layer are $\mathrm{U}$ and $\mathrm{Zr}$ in condition 1 ; the content of $\mathrm{U}$ is about $62.64 \%, \mathrm{Zr}$ is about $15.1 \%$, and stainless steel is very low, less than $5 \%$. Similar results are obtained in conditions 2 and 3 . The main elements in the 

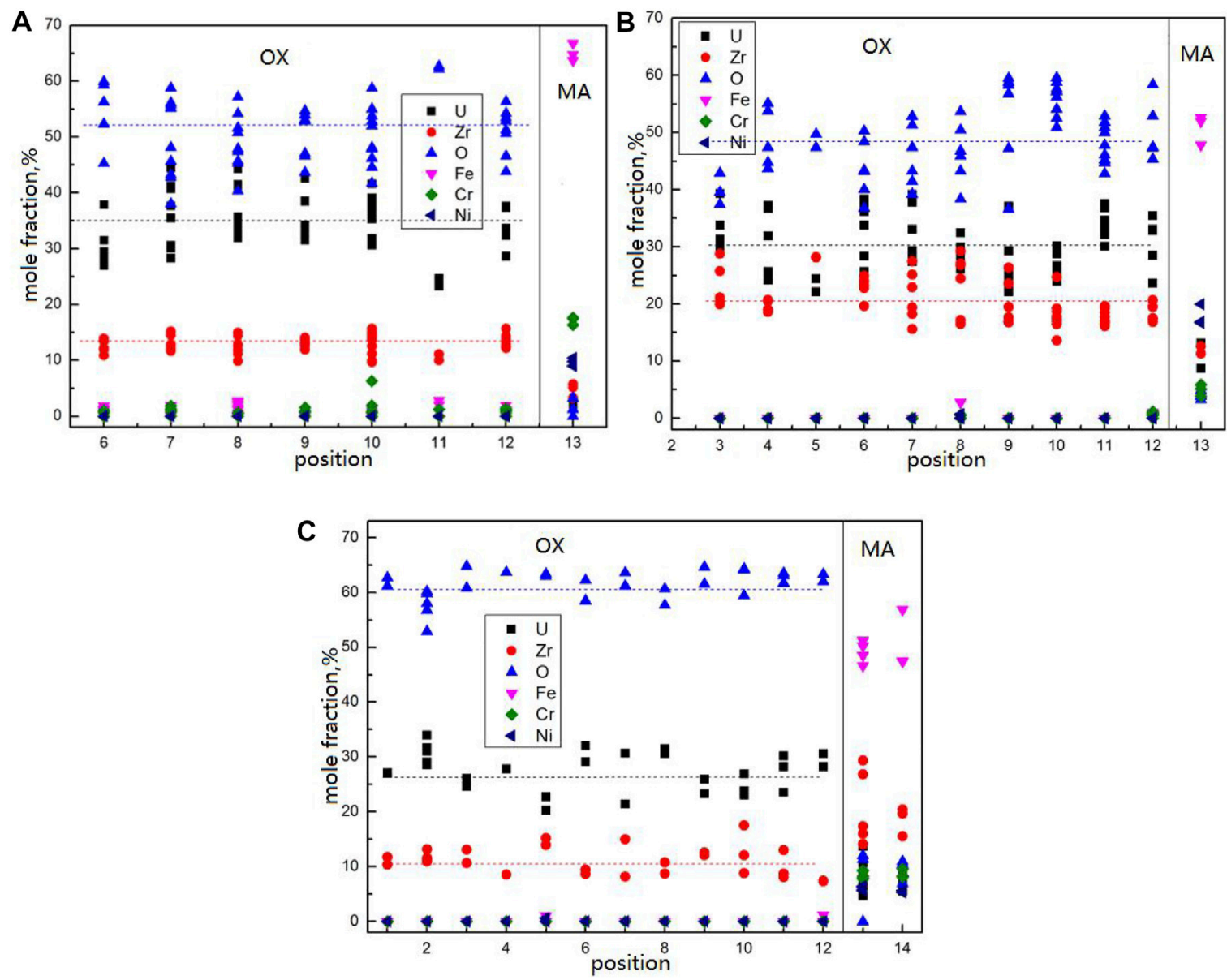

FIGURE 4 | Element distribution characteristics of the molten pool (A) 1, (B) 2, (C) 3.

\begin{tabular}{lcccc}
\hline \multicolumn{3}{l}{ TABLE 1 | Experimental condition. } & & \\
\hline Condition & $\mathbf{U} / \mathbf{Z r}$ & $\mathbf{C}_{\mathbf{n}}(\%)$ & $\mathbf{X}_{\mathbf{s s}}$ (\%) & Total(g) \\
\hline 1 & 1.5 & 100 & 10 & 5,000 \\
2 & 1.5 & 80 & 10 & 5,000 \\
3 & 1.5 & 40 & 10 & 5,000
\end{tabular}

metal layer are stainless steel, $\mathrm{Fe}, \mathrm{Cr}$, and $\mathrm{Ni}$; the content of $\mathrm{Fe}$ in the metal ball in condition one is as high as $62.2 \%$, and the contents of $U$ and $\mathrm{Zr}$ are very low. With the decrease of $\mathrm{C}_{\mathrm{n}}$, the Fe content in the metal layer decreases, and the contents of $U$ and $Z r$ increase. Under condition 3, the Fe content in the metal layer is about $34 \%$, while $U$ is about $21 \%$ and $\mathrm{Zr}$ is about $19.5 \%$. The increase of $U$ and $\mathrm{Zr}$ contents in the metal layer will increase the density of the metal layer, and the metal layer begins to move to the lower part of the molten pool.

Figure 4 shows the SEM/EDS test results of the experimental sample. It can be found that the elements of the oxide layer are mainly $\mathrm{U}, \mathrm{Zr}$, and $\mathrm{O}$, and there are certain differences in the content of elements at different positions. This may be that during the cooling process of the molten pool, the temperature gradient in the molten pool is large and segregation occurs, resulting in certain differences in the contents of $\mathrm{U}$ and $\mathrm{Zr}$ at different positions. Under condition 1 , the average content of $U$ in the oxide layer is about $35 \%$, the average content of $\mathrm{Zr}$ is about $14 \%$, and the average content of $\mathrm{O}$ is about $52 \%$, forming the oxide $(\mathrm{U}, \mathrm{Zr}) \mathrm{O}_{1.3}$. The content of stainless steel in the oxide layer is very low, basically below $3 \%$. Under condition 2 , the average content of $U$ in the oxide layer is about $32 \%$, the average content of $\mathrm{Zr}$ is about $20 \%$, and the average content of $\mathrm{O}$ is about $50 \%$. The oxide $(\mathrm{U}, \mathrm{Zr}) \mathrm{O}_{1}$ is formed. The oxide layer contains very little stainless steel, and the content is less than $2 \%$. Under condition 3, the average content of $U$ in the oxide layer is about $26 \%$, the average content of $\mathrm{Zr}$ is about $10 \%$, and the average content of $\mathrm{O}$ is about $60 \%$, forming the oxide $(\mathrm{U}$, $\mathrm{Zr}) \mathrm{O}_{1.7}$. The composition of stainless steel is not measured.

The metal ball has great hardness in condition 1 , and the main component is stainless steel $(\mathrm{Fe}, \mathrm{Cr}$, and $\mathrm{Ni})$, in which the content of Fe reaches about $65 \%$, and the content of $\mathrm{O}$ in the metal ball is very low, less than $3 \%$. In condition 2 , the main elements in the metal layer are also stainless steel ( $\mathrm{Fe}, \mathrm{Cr}$, and $\mathrm{Ni}$ ) and some $\mathrm{U}$ and $\mathrm{Zr}$, in which the content of $\mathrm{Fe}$ is about $50 \%$, the content of $\mathrm{Ni}$ is about $18 \%$, the content of $\mathrm{Cr}$ is about $5 \%$, the content of $\mathrm{U}$ is about $10 \%$, the content of $\mathrm{Zr}$ is about $12 \%$, and the content of $\mathrm{O}$ is about $4 \%$. In condition 3 , the metal layer is mainly composed of 
TABLE 2 | Elemental compositions of the corium samples measured using XRF (condition 1).

\begin{tabular}{|c|c|c|c|c|c|c|c|}
\hline Sample No & U (wt\%) & $\mathrm{Zr}(w t \%)$ & $\mathrm{Fe}(w t \%)$ & $\mathrm{Cr}(w t \%)$ & $\mathrm{Ni}(\mathbf{w t} \%)$ & Total (at\%) & $\mathrm{U} / \mathrm{Zr}$ \\
\hline $1-1$ & 0.17 & 56.40 & 7.50 & 7.60 & 0.34 & 72.03 & 0.00 \\
\hline $1-2$ & 20.30 & 54.50 & 0.62 & 1.00 & 0 & 76.39 & 0.14 \\
\hline $1-3$ & 42.90 & 2.50 & 25.30 & 6.10 & 1.70 & 78.50 & 6.56 \\
\hline $1-4$ & 74.60 & 4.70 & 3.00 & 2.60 & 0.13 & 85.07 & 6.07 \\
\hline $1-5$ & 15.20 & 59.90 & 0.57 & 0.64 & 0.05 & 76.31 & 0.10 \\
\hline $1-6$ & 57.00 & 17.10 & 4.80 & 3.40 & 0.12 & 82.43 & 1.27 \\
\hline $1-7$ & 63.20 & 14.70 & 3.50 & 1.70 & 0.13 & 83.16 & 1.64 \\
\hline $1-8$ & 60.50 & 16.40 & 4.30 & 1.30 & 0.61 & 83.09 & 1.41 \\
\hline $1-9$ & 63.90 & 15.40 & 2.70 & 1.50 & 0.12 & 83.67 & 1.59 \\
\hline $1-10$ & 64.00 & 13.50 & 3.90 & 2.00 & 0.30 & 83.69 & 1.81 \\
\hline $1-11$ & 63.00 & 16.40 & 2.40 & 1.60 & 0.16 & 83.45 & 1.47 \\
\hline $1-12$ & 66.90 & 12.20 & 3.40 & 1.70 & 0.13 & 84.31 & 2.10 \\
\hline $1-13$ & 5.60 & 1.30 & 62.20 & 2.50 & 12.60 & 84.20 & 1.65 \\
\hline
\end{tabular}

stainless steel, in which the content of $\mathrm{Fe}$ is about $48 \%$, the content of $\mathrm{Cr}$ is about $9 \%$, the content of $\mathrm{Ni}$ is about $5 \%$, the content of $\mathrm{U}$ is about $8 \%$, the content of $\mathrm{Zr}$ is about $20 \%$, and the content of $\mathrm{O}$ is about $7.5 \%$.

It can be found from conditions 1,2 , and 3 that when the $\mathrm{R}_{\mathrm{U} / \mathrm{Zr}}$ is unchanged and the stainless steel content is low, $C_{n}$ is the key factor for the stratification of the molten pool. When $C_{n}$ reaches $100 \%$, the molten pool will form a single layer of the oxide layer. With the reduction of $C_{n}$, the molten pool begins to appear in stratification, the area occupied by the metal layer increases, and the $\mathrm{U}$ and $\mathrm{Zr}$ contents in the metal layer increase, resulting in the increase of the density of the metal layer, and the metal layer begins to sink. The molten pool is turned from a metal layer on the upper oxide layer to a metal layer on the lower oxide layer.

\section{CONCLUSION}

In this study, the prototype materials are used to study the stratification morphology of the molten pool, and the following conclusions are obtained:

1) After the experiment, a complete corium ingot was formed. The molten corium mainly includes the upper crust, upper cavity, metal-rich layer, oxide-rich layer, side crust, and lower unmelted part;

2) With different melt composition, the layered structure of the molten pool is different. An obvious layered structure of the molten pool is observed. One layer is the bright layer, and the other is the dark layer;

\section{REFERENCES}

Almjashev, V. I., Granovsky, V. S., and Khabensk, V. B. (2018). Experimental Study of Transient Phenomena in the Three-Liquid Oxidic-Metallic Corium Pool[J]. Nucl. Eng. Des. 332, 31-37. doi:10.1016/j.nucengdes.2018.03.004

Asmolov, V., and Behbahani, A. (2000). CSNI/NEA RASPLAV Seminar, 14-15 November 2000. Munich, Germany.

Asmolov, V. G. (2004). in Main Results of the First Phase of MASCA Project (France: RRC Kurchatov Institute). Integrated Report.
3) The main elements in the oxidation layer of the molten pool are $\mathrm{U}, \mathrm{Zr}$, and $\mathrm{O}$, and the content of stainless steel is low. The main elements in the metal layer are stainless steel and contain a certain amount of $\mathrm{U}$ and $\mathrm{Zr}$ at the same time;

4) With the decrease in the $\mathrm{Zr}$ oxidation degree, the contents of $U$ and $\mathrm{Zr}$ in the metal layer of the molten pool increase, the density of the metal layer increases, and the molten pool will turn over.

\section{DATA AVAILABILITY STATEMENT}

The raw data supporting the conclusion of this article will be made available by the authors, without undue reservation.

\section{AUTHOR CONTRIBUTIONS}

$\mathrm{YaL}$ is responsible for experiment and data processing. HG is responsible for experimental design. $\mathrm{YH}$ is responsible for experimental operation. SY is responsible for sample testing. YoL is responsible for providing overall ideas. $\mathrm{YZ}$ is responsible for providing overall ideas.

\section{FUNDING}

This work was supported by the National Key Research and Development Program of China (2018YFB1900104). A part of this work was also supported by the China National Nuclear Corporation.

Bakardjieva, S., Barrachin, M., and Bechta, S. (2010). Improvement of the European Thermodynamic Database NUCLEA [J]. Prog. Nucl. Energ., 52 (1), 84-96. doi:10.1016/j.pnucene.2009.09.014

Bechta, S. V., and Khabensky, V. B. (2006). Corrosion of Vessel Steel during its Interaction with Molten Corium Part 2: Model Development. Nucl. Eng. Des. 236, 1362-1370. doi:10.1016/ j.nucengdes.2005.12.008

Bechta, S. V., and Khabensky, V. B. (2001). Experimental Studies of Oxidic Molten Corium-Vessel Steel Interaction. Nucl. Eng. Des. 210, 193-224. doi:10.1016/ s0029-5493(01)00377-6 
Bechta, S. V., Khabensky, V. B., and Granovsky, V. S. (2005). CORPHAD and METCOR ISTC projects[A]. The First European Review Meeting on Severe Accident Research [C]. France.

Bechta, S. V., and Granovsky, V. S. (2008). Corium Phase Equilibria Based on MASCA, METCOR and CORPHAD Results. Nucl. Eng. Des. 238, 2761-2771. doi:10.1016/j.nucengdes.2008.04.018

Chevalier, P-Y., Fischer, E., and Bertrand, C. (2004). Progress in the Thermodynamic Modelling of the O-U-Zr Ternary System[J]. ScienceDirect 28, 15-40. doi:10.1016/j.calphad.2004.03.005

Edwards, R. K., and Martin, A. E. (1966). Phase Relation in the Uranium-Uranium Dioxide System at High Temperature. Thermodynamics 69 (5), 2423.

Fukasawa, M., Shigeyuki, T., and Mitsuhiro, H. (2005). Development of Thermodynamic Database for U-Zr-Fe-O-B-C-FPs System[J]. Nuclear Science Technology 42 (8), 706-716. doi:10.1080/18811248.2004.9726440

Fukasawa, M., and Shigeyuki, T. (2012). Thermodynamic Analysis for Molten Corium Stratification Test MASCA with Ionic Liquid U-Zr-Fe-O-B-C-FPs Database[J]. Nucl. Sci. Techn. 44 (9), 1210-1219. doi:10.1080/ 18811248.2007.9711364

Guéneau, C., Dauvois, V., Pérodeaud, P., Gonella, C., and Dugne, O. (1998). Liquid Immiscibility in a (O,U,Zr) Model Corium. J. Nucl. Mater. 254, 158-174. doi:10.1016/s0022-3115(98)00002-6

Ikeuchi, H., Yano, K., and Washiya, T. (2020). Chemical Forms of Uranium Evaluated by Thermodynamic Calculation Associated with Distribution of Core Materials in the Damaged Reactor Pressure Vessel. J. Nucl. Sci. Techn. 57, 704-718. doi:10.1080/00223131.2020.1720844

Kang, K-H., and Park, R-J. (2014). An Experimental Study on Layer Inversion in the Corium Pool during Asevere Accident. Nucl. Eng. Des. 278, 163-170. doi:10.1016/j.nucengdes.2014.07.005

Pascal, P., Trillon, G. T., and Journeau, C. (2005). The $\mathrm{UO}_{2}-\mathrm{ZrO}_{2}$ System at High Temperature $(\mathrm{T}>2000 \mathrm{~K}$ ):importance of the Meta-Stable Phases under Severe Accident Conditions[J]. ScienceDirect 344, 259-264. doi:10.1016/ j.jnucmat.2005.04.052

Report of Japanese Government to the IAEA Ministerial Conference on Nuclear Safety (2011). The Accident at TEPCO's Fukushima Nuclear Power Stations - Jun(), Nuclear Emergency Response Headquarters. Vienna: Government of Japan.
Sang, M. A., Song, J. H., and Kim, J-Y. (2016). Experimental Investigation on Molten Pool Representing Corium Composition at Fukushima Daiichi Nuclear Power Plant[J]. J. Nucl. Mater. 478, 164-171. doi:10.1016/j.jnucmat.2016.06.011

Song, J., An, S., and Kim, J-Y. (2020). Morphology and Phase Distributions of Molten Core in a Reactor Vessel[J]. Nucl. Mater. 542 (15), 542. doi:10.1016/ j.jnucmat.2020.152471

Song, J., Min, B., and Hong, S. (2018). An Investigation on the Physical and Chemical Behaviors of Fuel Debris during Severe Accident Progression[J]. Prog. Nucl. Energ. 106, 345-356. doi:10.1016/j.pnucene.2018.02.028

Takano, M., Nishi, T., and Shirasu, N. (2014). Characterization of Solidified Melt Among Materials of $\mathrm{UO}_{2}$ Fuel and $\mathrm{B}_{4} \mathrm{C}$ Control Blade[J]. J. Nucl. Sci. Techn. 51 (7-8), 859-875. doi:10.1080/00223131.2014.912567

Theofanous, T. G., Liu, C., Additon, S., Angelini, S., Kymäläinen, O., and Salmassi, T. (1997). In-vessel Coolability and Retention of a Core Melt. Nucl. Eng. Des. 169, 1-48. doi:10.1016/s0029-5493(97)00009-5

Tsurikov, D. F., Strizhov, V. F., and Bechta, S. V. (2007). Integrated Report Main Resultsof the MASCA1 and 2 Projects (France: RRC Kurchatov Institute, Organization for Economic Cooperation and Development).

Conflict of Interest: The authors declare that the research was conducted in the absence of any commercial or financial relationships that could be construed as a potential conflict of interest.

Publisher's Note: All claims expressed in this article are solely those of the authors and do not necessarily represent those of their affiliated organizations, or those of the publisher, the editors, and the reviewers. Any product that may be evaluated in this article, or claim that may be made by its manufacturer, is not guaranteed or endorsed by the publisher.

Copyright $\odot 2021 \mathrm{Li}$, Gong, Hu, Yang, Li and Zan. This is an open-access article distributed under the terms of the Creative Commons Attribution License (CC BY). The use, distribution or reproduction in other forums is permitted, provided the original author(s) and the copyright owner(s) are credited and that the original publication in this journal is cited, in accordance with accepted academic practice. No use, distribution or reproduction is permitted which does not comply with these terms. 